\title{
Effect of E-cigarettes on Vapers' Physical and Mental Health
}

\author{
Xinyi Li \\ University of California, Santa Barbara, \#93107, USA. \\ *Corresponding author Email: xinyili@ucsb.edu
}

\begin{abstract}
Smoking is common in the US and can lead to adverse physical and mental health effects. For instance, smokers are highly vulnerable to chronic illnesses that increase the risk of mortality. The smoking rates have declined with time, but the use of e-cigarettes or vaping has been rising since it is considered an alternative to smoking. Vaping is advantageous since it does not burn tobacco like traditional cigarettes. However, the e-liquid in e-cigarettes contains nicotine that can adversely affect people's health. Therefore, using nicotine to overcome cigarette addiction is not appropriate to avoid smoking. The primary objective of this project is to examine the extent to which e-cigarettes can cause physical and mental health problems. Three databases that include Google Scholar, PubMed, and MEDLINE were searched. The results reveal that nicotine intake through e-cigarettes can increase the risk of developing hypertension, stroke, ADHD, depression, and anxiety. Overall, e-cigarettes seem to be a better alternative to smoking, but they can also cause physical and mental health issues.
\end{abstract}

Keywords: E-cigarettes, Vaping, Smoking, Chronic illnesses, Nicotine

\section{INTRODUCTION}

Smoking is one of the most common habits in the US and contributes immensely to adverse physical and mental health effects. About $14 \%$ of people aged 18 years and more smoked cigarettes in the US in 2019. These people are at risk of developing chronic illnesses. Chronic diseases increase the risk of death. Indeed, about one out of five deaths in the US are attributed to smoking [1]. Many people have been educated on the significance of avoiding smoking. Thus, the smoking rates have declined with time. For example, the smoking rate has reduced from $20.9 \%$ to $14.0 \%$ from 2005 to 2019 [1]. Overall, smoking affects all age groups and should be a priority concern for the healthcare system. While some people understand the adverse effects of smoking and intend to quit, they have resorted to using e-cigarettes to avoid the adverse effects of smoking.

While research has been done to provide the prevalence and the adverse effects of e-cigarette smoking, there is limited information on the extent to which vapers can develop complications. Therefore, people may not understand the degree to which they are at risk of developing health problems when they use e-cigarettes [2]. Therefore, there is a need for evidence on the extent to which e-cigarettes can cause adverse mental and physical health problems.

The purpose of this paper is to explain the adverse effect of e-cigarette smoking, examine the extent to which e-cigarettes can cause physical and mental health problems, including hypertension, heart failure, stroke and ADHD, search for evidence on how to prevent it, and discuss ways by which healthcare providers can reduce the vaping prevalence. Hope this research could help people or any readers to be alert about vaping and quit from it.

\section{MULTIPLE ADVERSE HEALTH EFFECTS OF E-CIGARETTES}

Vaping is used as an alternative to smoking for people who may not want to quit the habit. Vaping is advantageous, considering that it does not burn tobacco like traditional cigarettes. E-cigarettes deliver e-liquid, which is much less harmful than tobacco. This advantage has made the e-cigarettes popular, especially among people who are used to smoking. For instance, about 2.3\% of all adults in the US now use e-cigarettes, and the prevalence is rising [2]. In most cases, people experiment with e-cigarettes while still smoking traditional cigarettes to transition without issues. For instance, $39.1 \%$ 
of all smokers are using e-cigarettes [2]. An estimated $37.9 \%$ of all people who use e-cigarettes were former smokers [2]. Therefore, as the number of people who smoke traditional cigarettes reduces, the number of ecigarettes is expected to rise.

\subsection{The risk of developing hypertension}

Unfortunately, e-liquid contains nicotine that can adversely affect people's health. For instance, consuming nicotine leads to the production of dopamine, which necessitates the repetition of the behavior. Therefore, using nicotine to overcome cigarette addiction is not an appropriate approach to avoiding smoking. Currently, more than $2.3 \%$ of people are addicted to e-cigarettes. Once people become addicted, they can develop multiple adverse health effects [2]. For example, nicotine is usually deposited in the lumen of the blood vessels. The blood vessels narrow, leading to the blood resistance against them during blood flow. The cardiac muscles must pump harder to force the blood through the narrow blood vessels. Consequently, vapers develop hypertension.

It is proved that the intake of nicotine through ecigarettes can increase the risk of developing hypertension. For instance, $22 \%$ of people that take nicotine can develop hypertension [3]. Additionally, 10\% of people who inhale nicotine develop obesity, a risk factor for hypertension [3]. The hypertension prevalence among female users is $4.3 \%$ compared to males at $23.4 \%$. This data reflects a higher smoking rate among men than women. Most importantly, more than $50 \%$ of patients using nicotine are not tested for blood pressure. The current number of people with hypertension globally is 1.13 billion, but it is expected to rise to 1.5 billion, and the main contributory factor is smoking e-cigarettes. From the year 2000 to 2015, the rate of hypertension among smokers increased more than nonsmokers [4]. Most importantly, vaping can increase the systolic blood pressure by $9,6 \pm 4,1 \mathrm{mmHg}$. Vaping can also increase diastolic BP by $7.1 \pm 3.9 \mathrm{mmHg}$ [5]. Finally, it can increase the cardiac rate by heart rate by $16,4 \pm 13$ [5]. Overall, the data indicate that the use of e-cigarettes can increase the risk of developing hypertension.

\subsection{The risk of developing a stroke}

The continued intake of nicotine can increase the risk of developing a stroke, adversely affecting the smokers' physical health. For instance, instead of traditional cigarettes that increase carbon monoxide and reduce oxygen, e-cigarettes can cause vasculitis [2]. This problem can lead to the formation of a thrombus that can impair blood flow to vital organs, including the brain. For this reason, patients can develop a stroke. Patients may experience dizziness, walking difficulties, confusion, and weakness. Therefore, the avoidance of e-cigarettes can prevent a stroke and its associated adverse health effects.

Stroke develops to various conditions due to vaping. For example, $29 \%$ of people who use electronic cigarettes develop hypertension that can later cause a stroke [6]. The risk of developing a stroke increases by $8 \%$ among vapers aged 60 years and above. Additionally, $19 \%$ of people hat smoke e-cigarettes do not understand that stroke is preventable, and $38 \%$ do not know where the stroke occurs. Those who smoke 25 or more ecigarettes per week have a $3.7 \%$ higher of developing a stroke. The risk reduces to $2.2 \%$ among people who smoke 1-14 e-cigarettes. Most importantly, the stroke risk increases by $15 \%$ among younger people who use ecigarettes. Overall, avoiding e-cigarettes can reduce the stroke risk.

\subsection{The risk of developing a heart failure}

Using e-cigarettes can also increase the risk of heart failure. The exact statistics indicating the risk of heart failure do not exist. However, hypertension is the main cause of heart failure in people using e-cigarettes [7] [8]. Therefore, statistics on hypertension can be used to estimate the risk of heart failure.

If Vapers develop hypertension, they should quit smoking and undergo treatment of the disease, lest they develop complications. For example, the continued nicotine intake using e-cigarettes can further deposit nicotine in the blood vessels [2]. Vapers may experience a further increase in cardiac contractility. With time, the cardiac muscles may become tired, and vapers may develop heart failure, leading to other uncomfortable physical health effects. For example, vapers may develop breathing difficulties due to chest congestion. Vapers may also develop edema due to extracellular fluid deposition that occurs due to the heart failure pathology. Therefore, avoiding e-cigarettes can lower the risk of developing heart failure and its associated adverse health effects.

\subsection{Mental health problems}

Using e-cigarettes can improve mental health initially but later cause adverse mental health effects. For example, initially, vapers can experience improved mood and concentration. Nicotine may also reduce stress and relax muscles. However, if vapers do not use it regularly, they may experience withdrawal symptoms that require taking it regularly [2]. Some of the withdrawal symptoms include irritability, headaches, and feelings of sadness. People may also experience increased anxiety or fatigue. At night, vapers can experience insomnia. When they engage in any activity, they can experience poor concentration. People who use nicotine become dependent on it to improve their quality of life for all these reasons. 
Moreover, electronic cigarettes can increase the ADHD risk by $45 \%$. In other words, the attention deficit hyperactivity disorder symptoms positively correlate with e-cigarette use (rs from .16-.23, within time; rs from .13-.20, across time) [9]. Exact statistics on the effect of e-cigarettes on anxiety are unavailable. However, e-cigarette use is associated with anxiety and depression [10]. Overall, the findings demonstrate that ecigarettes cannot be used as an alternative approach to traditional smoking since it is addictive and can lead to various mental health problems.

\section{SUGGESTIONS ON THE HEALTH EDUCATION OF VAPING PREVENTION}

The use of e-cigarettes can contribute immensely to hypertensive disorders more than other conditions. Therefore, preventing vaping can significantly lower the vulnerability to hypertension. People addicted to vaping can be told that hypertension is the most common physical problem caused by the nicotine found in the ecigarette. For the reason, health education programs should be set, and they should contain information on how nicotine enters the vascular system and causes vasoconstriction. People addicted to e-cigarettes should understand how reduced lumen of blood vessels increases intravascular resistance, leading to hypertension. This information can help them understand the risks to which they are exposed, leading to compliance with the health education recommendations to avoid vaping. According to the provided statistics, reducing the adverse physical effects of hypertension should be prioritized. For instance, vaping can increase systolic blood pressure by $9.6 \pm 4.1 \mathrm{mmHg}$ [3]. Ecigarettes also increase the heart rate by $16.4 \pm 13$. Therefore, the vascular system and the cardiac muscles can experience significant changes in function due to nicotine that comes from e-cigarettes. Overall, health education for patients taking e-cigarettes should be done, focusing on the increased risk of hypertension development.

Vaping addicts should be informed that the continued intake of nicotine in e-cigarettes can lead to heart failure. The identified results do not provide the exact heart failure statistics that develop directly from taking ecigarettes. However, the identified evidence indicates that the heart failure risk increases among patients with hypertension using e-cigarettes [7] [8]. Healthcare providers should educate patients on various health promotion strategies to prevent this complication, including avoiding vaping. For example, inadequate physical activity can exacerbate hypertension and its effects [7]. Therefore, patients using e-cigarettes should avoid the habit and engage in lifestyle changes that involve improved physical activity to improve their health. Most importantly, the evidence indicates that hypertension prevalence can increase due to an increase in vaping cases [7]. Therefore, programs aimed at reducing the use of e-cigarettes can help reduce hypertension prevalence and its complications, including heart failure.

Vaping addicts should also understand that the habit can cause a stroke. Health education should contain valid information on how a stroke develops. For example, vaping addicts should understand that a stroke occurs due to an insufficient blood supply to the brain [6]. They should also understand that using e-cigarettes can cause inflammation of the vascular system and impair blood flow to the brain. Information on how clotting can form in the inflamed blood vessels and increase the risk of a stroke should also be integrated into the educational intervention. Overall, vaping addicts should understand that the avoidance of vaping is a significant approach to reducing a stroke. Programs to reduce the stroke prevalence due to vaping should be intensified among young people. The evidence indicates that the stroke risk increases by $15 \%$ among younger people who use ecigarettes [6]. For this reason, all teenagers using ecigarettes should be encouraged to quit vaping to improve their health outcomes for the long term.

Vaping addicts should understand their risk of developing mental health issues to find a reason to quit the habit. Once they understand the risks, they should be encouraged to cease using e-cigarettes. The encouragement to quit vaping can improve mental health, just like it can improve physical health. For instance, they should understand that the most common outcome of vaping, especially among students, is ADHD, whose risk is $45 \%$ [9]. This figure is nearly half and demonstrates increased risk, which patients should understand. Therefore, recommending vaping cessation can reduce the risk of ADHD by $45 \%$. Other risks that vaping addicts should understand are anxiety and depression. They can understand that by avoiding the problem, they can also avoid these health issues. Initially, vaping can enhance concentration and mood [7]. The adverse effects occur as withdrawal symptoms. For this reason, it may be challenging to explain to e-cigarette addicts that the habit can lead to mental health issues and concentration problems. They should understand that they can avoid multiple mental health issues that either occur due to smoking e-cigarettes or by withdrawing from them. Overall, health education programs should help ecigarette addicts understand that they can develop withdrawal symptoms, but such outcomes are a way to recovery. Nurses in healthcare settings should collaborate with public health professionals to initiate educational programs. By doing so, they can limit curative care and enhance preventive care within communities.

\section{CONCLUSION}

Although the efforts to encourage smoking cessation 
are productive, smokers are not quitting but using ecigarettes to avoid smoking traditional cigarettes. The prevalence of e-cigarette smoking is rising and increasing the risk of developing physical and mental health issues. For instance, e-cigarettes contain nicotine, which can cause vasoconstriction and lead to hypertension. The continued use of e-cigarettes can exacerbate hypertension and lead to heart failure. The inflammation of blood vessels that occurs due to ecigarette smoking can cause a stroke. The stroke occurrence risk increases when people begin vaping at a young age. For this reason, people should be encouraged to avoid e-cigarettes to reduce the risk of hypertension, heart failure, and stroke. People who decide to stop vaping may develop ADHD, depression, and anxiety. However, these symptoms can subside since they only occur due to withdrawal. Overall, people's willingness to avoid vaping can reduce the risk of mental and physical health issues. More researches should be done focusing on vapers' psychological movements and their intentions to have e-cigarettes so that researchers can be more effective to conclude the most efficient way to help people quit vaping.

\section{REFERENCES}

[1] Centers for Disease Control and Prevention, Current Cigarette Smoking Among Adults in the United States, 10-December-2020, Doi: https://www.cdc.gov/tobacco/data_statistics/fact_s heets/adult_data/cig_smoking/index.htm.

[2] Mayer, Margaret, Carolyn Reyes-Guzman, Rachel Grana, Kelvin Choi, and Neal D. Freedman. "Demographic characteristics, cigarette smoking, and e-cigarette use among US adults." JAMA Network Open 3, no. 2020, 10: e2020694-e2020694. Doi:

https://doi.org/10.1001/jamanetworkopen.2020.206 94.

[3] S.N. Walekhwa, A. Kisa, Tobacco use and risk factors for hypertensive individuals in Kenya. Healthcare (Basel, Switzerland), 9(5), 2021, 591.

Doi: https://doi.org/10.3390/healthcare9050591.

[4] H. Andriani, R.I. Kosasih, S. Putri, H.W. Kuo, Effects of changes in smoking status on blood pressure among adult males and females in Indonesia: A 15year population-based cohort study. BMJ Open, 10(4), 2020, e038021. Doi: https://doi.org/10.1136/bmjopen-2020-038021.

[5] G. Crippa, M. Bergonzi, E. Bravi, V. Balordi, A. Cassi, Effect of electronic cigarette smoking on blood pressure in hypertensive patients. evaluation by non-invasive continuous ambulatory blood pressure measurement. Journal of Hypertension, 36, e4,

2018.

Doi:

https://doi.org/10.1097/01.hjh.0000538974.96760.

a3.

[6] A.P. Klein, K. Yarbrough, J.W. Cole, Stroke, Smoking and Vaping: The No-Good, the Bad and the Ugly. Annals of Public Health and Research, 8(1), 2021, $1104 . \quad$ Doi: https://www.ncbi.nlm.nih.gov/pmc/articles/PMC83 15328/\#R87.

[7] M. Peruzzi, G. Biondi-Zoccai, R. Carnevale, E. Cavarretta, G. Frati, F. Versaci, Vaping cardiovascular health risks: An updated umbrella review. Current emergency and hospital medicine reports, 2020, pp. 1-7. Doi: https://doi.org/10.1007/s40138-020-00219-0.

[8] H. Shi, X. Fan, A. Horton, S.T. Haller, D.J. Kennedy, I.T. Schiefer, L. Dworkin, C.J. Cooper, J. Tian, The Effect of electronic-cigarette vaping on cardiac function and angiogenesis in mice. Scientific Reports, 9(1), 2019, $4085 . \quad$ Doi: https://doi.org/10.1038/s41598-019-40847-5.

[9] N.I. Goldenson, R. Khoddam, M.D. Stone, A.M. Leventhal, Associations of ADHD symptoms with smoking and alternative tobacco product use initiation during adolescence. Journal of Pediatric Psychology, 43(6), 2018, pp. 613-624. Doi: https://doi.org/10.1093/jpepsy/jsx153.

[10] M.R. Dvorsky, J.M. Langberg, Cigarette and ecigarette use and social perceptions over the transition to college: The role of ADHD symptoms. Psychology of Addictive Behaviors, 33(3), 2019, pp. 318-330. Doi: https://doi.org/10.1037/adb0000450.

[11] M.J. Zvolensky, N.A. Mayorga, L. Garey, Worry, anxiety sensitivity, and electronic cigarettes among adults. Psychiatry Research, 269, 2019, pp. 321-327. Doi:

https://doi.org/10.1016/j.psychres.2018.08.087.

[12] O.H. Obisesan, M. Mirbolouk, A.D. Osei, O.A. Orimoloye, S. Uddin, O. Dzaye, O. El Shahawy, M. Al Rifai, A. Bhatnagar, A. Stokes, E.J. Benjamin, A.P. DeFilippis, M.J. Blaha, Association between ecigarette use and depression in the behavioral risk factor surveillance system, 2016-2017. JAMA Network Open, 2(12), 2019, e1916800. Doi: https://doi.org/10.1001/jamanetworkopen.2019.168 00 .

[13] S.N. Walekhwa, A. Kisa, Tobacco use and risk factors for hypertensive individuals in Kenya. Healthcare (Basel, Switzerland), 9(5), 2021, 591.

Doi: https://doi.org/10.3390/healthcare9050591. 
[14] M. Peruzzi, G. Biondi-Zoccai, R. Carnevale, E. Cavarretta, G. Frati, F. Versaci, Vaping cardiovascular health risks: An updated umbrella review. Current emergency and hospital medicine reports, 2020, pp. 1-7. Doi: https://doi.org/10.1007/s40138-020-00219-0.

[15] G. Crippa, M. Bergonzi, E. Bravi, V. Balordi, A. Cassi, Effect of electronic cigarette smoking on blood pressure in hypertensive patients. evaluation by non-invasive continuous ambulatory blood pressure measurement. Journal of Hypertension, 2018, 36, e4. Doi: https://doi.org/10.1097/01.hjh.0000538974.96760. a3.

[16] N.I. Goldenson, R. Khoddam, M.D. Stone, A.M. Leventhal, Associations of ADHD symptoms with smoking and alternative tobacco product use initiation during adolescence. Journal of Pediatric Psychology, 43(6), 2018, pp. 613-624. Doi: https://doi.org/10.1093/jpepsy/jsx153. 